\title{
Dissipation, Noise, and Vacuum Decay in Quantum Field Theory
}

\author{
Esteban Calzetta \\ Departamento de Física, Universidad de Buenos Aires, Ciudad Universitaria, 1428 Buenos Aires, Argentina
}

\begin{abstract}
Albert Roura and Enric Verdaguer*
Departament de Física Fonamental, Universitat de Barcelona, Avinguda Diagonal 647, 08028 Barcelona, Spain
\end{abstract}

(Received 25 April 2001; published 18 December 2001)

\begin{abstract}
We study the process of vacuum decay in quantum field theory focusing on the stochastic aspects of the interaction between long- and short-wavelength modes. This interaction results in a diffusive behavior of the reduced Wigner function describing the state of long-wavelength modes, and thereby to a finite activation rate even at zero temperature. This effect can make a substantial contribution to the total decay rate.
\end{abstract}

DOI: 10.1103/PhysRevLett.88.010403

PACS numbers: 03.70.+k, 05.40.Ca, 11.10.-z

In this Letter we investigate how stochastic interactions between long- and short-wavelength modes affect vacuum decay in scalar quantum field theory. Two of the present authors have already considered the relevance of stochasticity in the context of the creation from nothing of the Universe [1]. Our analysis of that problem led to the conclusion that the noise-induced transition amplitude was actually larger than the usual quantum estimates [2]. However, it remained unclear whether the relevance of stochasticity for the full decay amplitude was a peculiarity of gravitationally bound systems, or rather a generic feature of vacuum decay in field theory. The results we discuss here point quite conclusively in the second direction. In pursuit of clarity, we omit most of the technical details, which shall be reported in separate publications $[3,4]$.

As a simple nongravitational example, let us consider a self-interacting scalar field $\Phi$ in Minkowski spacetime. The classical action is

$$
S_{\mathrm{ren}}[\Phi]=-\frac{1}{2} \int d^{4} x\left(\partial \Phi \partial \Phi+M^{2} \Phi^{2}-\frac{1}{3} g \Phi^{3}\right) .
$$

Although we keep $\hbar$ explicit we set $c=1, M$ has units of length $^{-1}, \Phi$ has units of $M \sqrt{\hbar}$, and $g$ of $M / \sqrt{\hbar}$. For simplicity, we assume that renormalization has already been carried out and that Eq. (1) is a good description of the relevant dynamics. This means that the parameters $M^{2}$ and $g$ may well be renormalization point dependent; in any case, any such dependence will be taken as given. This model may be considered as the limiting case of the class of models studied by Baacke and Kiselev [5], when the coupling in the quartic self-interaction is very small.

We are concerned with situations where the potential displays a local minimum, separated from the absolute minimum by a potential barrier. A system of few degrees of freedom, prepared in a false vacuum state within a potential well, may decay in essentially two different ways, namely, by tunnel effect, that is, going through the barrier in a classically forbidden trajectory, or else by activation, that is, jumping over the barrier [6,7]. In systems with few degrees of freedom, there must be an external agent, typically a thermal source, for activation to be possible. Activation results from the system being driven by noise originating in the source.

In either case, the decay probability follows the Arrhenius law $P \sim A e^{-B}$. In the tunnel effect, $B=S_{E} / \hbar$, where $\hbar$ is Planck's constant and $S_{E}$ is the action for the trajectory which goes under the barrier in Euclidean time [8]. In activation, $B=V_{s} / k_{B} T$, where $k_{B}$ is Boltzmann's constant, $T$ is the temperature, and $V_{s}$ is the height of the free energy barrier measured from the false vacuum [9]. We can see that activation disappears as $T \rightarrow 0$.

Our thesis is that in field theories there is a phenomenon similar to activation, even in the absence of an external environment and, most remarkably, that this simulated activation contributes to vacuum decay probability even at zero temperature. This phenomenon exists because, while vacuum decay concerns mainly the long-wavelength modes in the field, these modes evolve in the environment provided by the short-wavelength ones. Because of the time dependence of the long-wavelength modes, even if the short-wavelength modes were initially prepared in their vacuum states, these will evolve into coherent superpositions of many particle states. The energy to create these particles is provided by the long-wavelength modes. On the other hand, it is not possible to predict the exact number of particles to be created. For Bose-Einstein statistics, for example, if $N$ particles are created in the mean, then the dispersion in this number is of order $\sqrt{N(N+1)}$, and it is never negligible.

Therefore, we find a dissipative term in the dynamics of the long-wavelength modes, representing the energy transfer towards the short-wavelength modes, but also a stochastic element, related to the fluctuations in the energy flux. These two terms are related to each other through the fluctuation-dissipation theorems. We must stress that the presence of one of them implies the presence of the other as well. Unlike in Kramers' activation, this environment is intrinsic to the system. We should point out that 
because of this same reason we are not allowed to prescribe the characteristics of noise and dissipation independently of the system dynamics. This means that it is not possible in general to assume Ohmic dissipation or white noise [10].

More concretely, tunneling occurs in models where the system may be trapped into a metastable state, which is separated from the basin of attraction of the true vacuum by a potential barrier. There is a saddle point on this barrier, representing the critical bubble, and most of the tunneling dynamics is concerned with motion along the most likely escape path [11], which goes through the saddle in the direction of steepest descent. It is possible to identify a few degrees of freedom which parametrize the different configurations on this path; the remaining (infinite) degrees of freedom describe deviations from the most likely escape route. In the conventional approaches to tunneling, the role of these fluctuations is downplayed: they renormalize the action for the few distinguished parameters and provide a prefactor which, after the contributions to the effective potential have been included in the exponential, is of order one [12]. We intend to focus on the backreaction of the fluctuations away from the most likely escape path on the quantum dynamics of the critical bubble; for this purpose, we borrow tools from quantum open systems theory, by considering the few distinguished degrees of freedom as a system interacting with the environment provided by the transversal fluctuations.

The technical complexity of the problem increases sharply with the amount of information one wishes to retain within the system. For example, one may parametrize the most likely escape path following Ref. [13]. As shown in this reference (and earlier in Ref. [14]), a time dependent bubble excites the degrees of freedom in the environment, thus setting up the kind of dynamical interplay we wish to analyze (see also Ref. [15]). To be able to focus on the new (dynamical) aspects of the problem, over and above its geometrical aspects, we adopt an intentionally simplified parametrization of the most likely escape path which retains the essentials of the physics involved.

Let us return to the scalar field theory above. The potential $V[\phi]=\frac{1}{2} M^{2} \phi^{2}-\frac{1}{6} g \phi^{3}$ has a stable fixed point at $\phi=0$ and an unstable fixed point at $\phi=\phi_{s}=2 g^{-1} M^{2}$. The former corresponds to zero energy, and the latter to $E=E_{s}=V M^{2} \phi_{s}^{2} / 6$ in a volume $V$. For intermediate energies, we may have bound and unbound states. They are separated by a potential barrier, which at zero energy extends from $\phi=0$ to $\phi=\phi_{\text {exit }}=3 \phi_{s} / 2$. To identify the relevant modes, we observe that if we consider fluctuations around the unstable fixed point $\phi_{s}$, then modes with wave number $k>M$ are stable. The relevant modes, which partake in the tunneling process, have $k<M$ [16]. We therefore write the field as $\Phi=\phi+\varphi$, where the first term contains only modes with $k<M$, and the second term contains the short wavelengths; $\phi$ is our system.
In other words, the field $\phi$ represents the average of the full field $\Phi$ over volumes of order $M^{-3}$. By construction, $\phi$ is slowly varying in space; it is technically simplest to handle it as if it were actually spatially homogeneous. We therefore regard the configurations along the most likely escape path as a sequence of "top hat" field configurations, parametrized by a single degree of freedom $\phi(t)$, representing the field amplitude within a domain of size $M^{-1}$, outside of which the system field vanishes. The center of mass coordinates of the "hat" may be treated as collective coordinates in the usual way and do not affect our results [17].

If the quantum state of the full field is described by a density matrix $\rho\left(\phi, \varphi, \phi^{\prime}, \varphi^{\prime}, t\right)$, the state of the $\phi$ field is described by the reduced density matrix $\rho_{r}\left(\phi, \phi^{\prime}, t\right)=\int d \varphi \rho\left(\phi, \varphi, \phi^{\prime}, \varphi, t\right)$, or equivalently by the reduced Wigner function

$$
f(\phi, p, t)=\int \frac{d u}{2 \pi \hbar} e^{-i p u / \hbar} \rho_{r}\left(\phi+\frac{u}{2}, \phi-\frac{u}{2}, t\right) .
$$

To second order in $g$ and leading order in $\hbar, f$ evolves according to [4]

$$
\frac{\partial f}{\partial t}=\left\{H_{s}, f\right\}+\frac{\partial}{\partial p}(\Gamma f+\hbar\{N, f\})-\frac{g \hbar^{2}}{24} \frac{\partial^{3} f}{\partial p^{3}},
$$

where the curly brackets are Poisson brackets, $H_{s}=$ $\dot{\phi}^{2} / 2+V(\phi), \quad \Gamma=\int d t^{\prime} H\left(t-t^{\prime}\right) \phi\left(t^{\prime}\right), \quad$ and $\quad N=$ $\int d t^{\prime} N\left(t-t^{\prime}\right) \phi\left(t^{\prime}\right)$. The kernels $H$ and $N$ represent the effects of dissipation and noise, respectively. They come from the quadratic part of the FeynmanVernon influence action [18]. Computing the influence functional requires handling formally infinite quantities (and in our case, also a linear term in $\phi$ ); regularization and renormalization leave a finite residuum, which are the one-loop correction to the effective potential and a finite wave function renormalization. As we have already remarked, we assume that these corrections are already included in Eq. (1). This is sensible because, although they may be quantitatively important, they do not affect the nature of the problem [12].

Our approach to Eq. (3) will be the following: It is clear that if only the first term of the right-hand side is kept, the equation reduces to the classical transport equation and there is no tunneling. Retaining the first and last terms on the right-hand side is equivalent to writing a Schrödinger equation for the wave function of the homogeneous mode, as if it were a closed system. Since this is a onedimensional problem, the tunneling rate may be computed either by the instanton or the WKB method, which are known to be equivalent in this case. We wish to know if the middle (backreaction) term makes a substantial contribution to the total rate. With this strategy in mind we discard the third contribution to the right-hand side in 
Eq. (3), assuming implicitly that the backreaction term is dominant (see below). It then reduces to Kramers' equation which may be seen [4] to describe the evolution of an ensemble of points evolving according to the Langevin equation: $d \phi / d t=p(t)$,

$$
\frac{d p}{d t}(t)=-V^{\prime}[\phi(t)]+\int d t^{\prime} H\left(t-t^{\prime}\right) \phi\left(t^{\prime}\right)+\xi,
$$

with initial conditions $\left(\phi_{i}, p_{i}\right)$ weighted according to the initial Wigner function, and Gaussian noise characterized by $\left\langle\xi(t) \xi\left(t^{\prime}\right)\right\rangle=\hbar N\left(t-t^{\prime}\right)$. Although this representation of the dynamics has an important heuristic value, only the reduced Wigner function $f$ has a direct physical meaning, and it does not allow an interpretation as a classical distribution function in general (it is not generally positive definite)

We are interested in the weak dissipation limit, as discussed by Kramers [6], when the relaxation time is long compared with the classical period of motion. On the other hand, the memory time in the integrals in Eq. (3) is determined by the frequencies in the environment, which are large with respect to the dominant frequencies in the system. Thus we are allowed to (and, in a formal expansion in powers of $\hbar$, we must) use solutions to the classical equations of motion within the memory terms; these solutions may be written down explicitly in terms of elliptic functions. This is a less drastic approximation than the Markovian one discussed in Ref. [19]. We also neglect transient terms (or in other words, we assume $t \gg M^{-1}$ ); this means that we can take the lower limit of the time integrals in Eq. (3) as $t=-\infty$. In the weak dissipation limit the reduced Wigner function depends only on the action variable $J=\frac{1}{2 \pi} \oint d \phi p$, and, averaging over angles, Kramers' equation reduces to a onedimensional Fokker-Planck equation $\partial f / \partial t+\partial \bar{\Phi} / \partial J=$ 0 , where $\bar{\Phi}=-\Theta \Omega^{1} \partial f / \partial J-\Lambda f$ is the flux, and $\Omega=\Omega(J)$ is the frequency of the corresponding classical motion [20]. The point of this analysis is that it is possible to derive explicit expressions for the coefficients $\Theta$ and $\Lambda$ in the Fokker-Planck equation [1,4]. Near the value $J_{s}$ of the action variable at the separatrix (that is, the limiting trajectory which connects to the unstable equilibrium point) these have finite values, while as $J \rightarrow 0$ they go to zero as $E^{3}$, where $E=H_{s}(J)$. In the thermal activation problem one finds an identical equation, but the coefficients decay linearly on $E$ [6].

The weakness of noise and dissipation in our (vacuum decay) problem reflects the origin of these effects in particle creation. Since particles are created in pairs, there is a threshold for particle creation at frequency $\omega \sim 2 M$. At low energy, classical motion is mainly harmonic with frequency $M$ for small oscillations around the metastable minimum; hence particle creation is weak. It never actually vanishes, though, because at any finite energy there is a small deviation from harmonic motion. The amplitude of the component with frequency $n \Omega$ decays as $E^{n}$ as $E \rightarrow 0$, which is enough to trigger particle creation [21].

The Fokker-Planck equation describes an initial value problem subject to nontrivial boundary conditions at $J=$ 0 and $J=J_{s}$. These are vanishing flux $\bar{\Phi}=0$ at $J=0$, and vanishing probability $f=0$ at $J=J_{s}$. The linear operator $L$ which is defined by $L f=\partial \bar{\Phi} / \partial J$ is selfadjoint with respect to an adequate inner product [4], and the equation may be solved by an expansion in normal modes in the usual way. A general solution is reconstructed as a superposition of modes $f_{r}$ decaying as $\exp (-r t)$. For a given $r, f_{r}$ oscillates as $J \rightarrow 0$, and the modes must be subject to a continuum normalization, as in the usual treatment of the WKB wave function in quantum mechanics [22]. The result is that, given any smooth initial condition with mean energies of the order of the false vacuum energy $\hbar M / 2$, the persistency amplitude $P(t)=2 \pi \int d J f(J, t)$ decays exponentially with a constant $\lambda$ for $\lambda t \gtrsim 1$, turning to $1 / t$ for longer times (this crossover is also observed in the usual tunneling amplitude [23]). The constant is [4] $\lambda \approx \Delta \exp \left\{-\int d E \frac{\Lambda}{\Theta}(E)\right\} \sim \Delta \exp \left\{-a \frac{M^{2}}{\hbar g^{2}}\right\}$, where $\Delta$ is of order 1 , and $a \sim 0.2$. By contrast, the tunneling amplitude, in the corresponding approximation of only considering the homogeneous mode, yields a similar formula, but with $a \sim 4.8$ [8]. We can see that, in this case, the zero temperature activation rate is higher than the tunneling amplitude by an order of magnitude in the exponent.

We point out that the fact that in our example the activation amplitude is actually larger than the tunneling amplitude is model dependent. Roughly speaking, low and broad barriers favor activation, while high and narrow barriers favor tunneling. We also stress that these results must be considered as preliminary, pending a more satisfactory parametrization of the system and therefore a more realistic modelization of the system-bath interaction. It is safe to conclude, however, that activation should not be discarded a priori but rather should be counted on as a potentially significant contribution to the overall decay amplitude.

Note that we reach a different conclusion to that of Ref. [24], where dissipation suppresses tunneling. However, a direct comparison between the two analyses cannot be done as the two models differ essentially in the coupling with the environment degrees of freedom, which is quadratic in our case. Our analysis is closer to that of Ref. [25], and it is certainly compatible with their conclusions; see also Ref. [26].

Still, we stress that we should not expect a similar behavior in systems with few degrees of freedom. The fact that in our problem the environment actually contained a large enough number of degrees of freedom as to represent a continuum for all practical purposes is essential to provide a suitable driving force. If some frequency intervals were lacking, then there would arise islands of stability where no resonance is strong enough to move the system forward. These islands would act as absolute barriers to noise-induced decay, or at least would depress 
the noise induced amplitude much below the tunneling estimates.

In conclusion, we have shown that vacuum decay in field theory is qualitatively different from the same process in systems with few degrees of freedom, because the former are intrinsically open systems. Interaction between longand short-wavelength modes induce a stochastic dynamics for the former and results in activation even at zero temperature.

We thank Leticia Cugliandolo, Bei-Lok $\mathrm{Hu}$, Jaume Garriga, Rodolfo Id Betan, and Jorge Kurchan for many illuminating discussions. This work has been partially supported by Fundación Antorchas under Grant No. A-13622/1-21. E.C. acknowledges support from Universidad de Buenos Aires, CONICET, Fundación Antorchas, and the ANPCYT through Project No. PICT99 03-05229. A. R. and E. V. have also been supported by the CICYT Research Project No. AEN98-0431. E. V. also acknowledges support from the Spanish Ministry of Education under FPU Grant No. PR2000-0181 and the University of Maryland for hospitality.

*Also at Institut de Física d'Altes Energies (IFAE), Barcelona, Spain.

[1] E. Calzetta and E. Verdaguer, Phys. Rev. D 59, 083513 (1999); E. Calzetta, Int. J. Theor. Phys. 38, 2755 (1999).

[2] A. Vilenkin, Phys. Lett. 117B, 25 (1982); Phys. Rev. D 27, 2848 (1983); 30, 509 (1984).

[3] E. Calzetta, A. Roura, and E. Verdaguer, quant-ph/ 0011097.

[4] E. Calzetta, A. Roura, and E. Verdaguer, hep-ph/0106091.

[5] J. Baacke and V. G. Kiselev, Phys. Rev. D 48, 5648 (1993).

[6] H. Kramers, Physica (Utrecht) 7, 284 (1940).

[7] J. Langer, Ann. Phys. (N.Y.) 54, 258 (1969).

[8] A. D. Linde, Nucl. Phys. B216, 421 (1983); J.-L. Gervais and B. Sakita, Phys. Rev. D 16, 3507 (1977); H. De Vega, J.-L. Gervais, and B. Sakita, Nucl. Phys. B139, 20 (1978);
S. Coleman, Phys. Rev. D 15, 2929 (1977); C. G. Callan and S. Coleman, Phys. Rev. D 16, 1762 (1977); S. Coleman, Aspects of Symmetry (Cambridge University Press, Cambridge, U.K., 1985).

[9] P. Hanggi, P. Talkner, and M. Borkovec, Rev. Mod. Phys. 62, 251 (1990); I. Affleck, Phys. Rev. Lett. 46, 388 (1981).

[10] W. Horsthemke and R. Lefever, Noise-Induced Transitions (Springer, Berlin, 1984); M. Griffoni and P. Hanggi, Phys. Rep. 304, 229 (1998).

[11] T. Banks, C. Bender, and T. Wu, Phys. Rev. D 8, 3346 (1973).

[12] M. Gleiser, G. C. Marques, and R. O. Ramos, Phys. Rev. D 48, 1571 (1993); J. Baacke and S. Junker, Phys. Rev. D 50, 4227 (1994); A. Surig, Phys. Rev. D 57, 5049 (1998).

[13] T. Vachaspati and A. Vilenkin, Phys. Rev. D 43, 3846 (1991).

[14] V. Rubakov, Nucl. Phys. B245, 481 (1984).

[15] T. Tanaka, M. Sasaki, and K. Yamamoto, Phys. Rev. D 49, 1039 (1994); S. Alamoudi et al., Phys. Rev. D 60, 125003 (1999).

[16] D. Boyanovsky and C. Aragao de Carvalho, Phys. Rev. D 48, 5850 (1993); D. Boyanovsky et al., Nucl. Phys. B441, 595 (1995); B441, 609 (1995).

[17] R. Rajaraman, Solitons and Instantons (Elsevier, Amsterdam, 1982).

[18] E. Calzetta and B. L. Hu, Phys. Rev. D 40, 656 (1989).

[19] A. Berera and R. Ramos, Phys. Rev. D 63, 103509 (2001).

[20] H. Risken, The Fokker-Planck Equation (Springer-Verlag, Berlin, 1989); C. W. Gardiner, Handbook of Stochastic Methods (Springer-Verlag, Berlin, 1994).

[21] E. Calzetta and B. L. Hu, Phys. Rev. D 55, 3536 (1997); D. Boyanovsky, H. J. de Vega, R. Holman, and J. F. J. Salgado, Phys. Rev. D 54, 7570 (1996).

[22] L. Landau and E. Lifshitz, Quantum Mechanics, NonRelativistic Theory (Pergamon, London, Addison-Wesley, Reading, MA, 1958).

[23] L. Khalfin, J. Exp. Theor. Phys. 33, 1371 (1957) [Sov. Phys. JETP 6, 1053 (1958)].

[24] A. Caldeira and A. Leggett, Ann. Phys. (N.Y.) 149, 374 (1983).

[25] R. Bruinsma and P. Bak, Phys. Rev. Lett. 56, 420 (1986).

[26] K. Fujikawa et al., Phys. Rev. B 46, 10295 (1992). 